\title{
Delayed diagnosis of juvenile myoclonic epilepsy
}

\author{
R A Grünewald, E Chroni, C P Panayiotopoulos
}

\begin{abstract}
Fifteen cases of juvenile myoclonic epilepsy (JME) were identified from one hundred and eighty consecutive patients referred to a new epilepsy clinic at St Thomas' Hospital between April 1989 and December 1990 , a prevalence of $8 \cdot 3 \%$. Of these, only one was referred with a putative diagnosis of JME. Diagnosis of the other patients on referral included "epilepsy", "grand mal", "temporal lobe epilepsy", "photoconvulsive epilepsy" and "alcohol-induced epilepsy". At least 11 of the 15 patients had been seen by a neurologist in the United Kingdom before referral. Definitive diagnosis was delayed by a mean of 14.5 years. In seven patients inappropriate anticonvulsants had been prescribed. Control of seizures was improved in most patients after diagnosis. Factors responsible for the delay in diagnosis include lack of familiarity with the syndrome, failure to elicit a history of myoclonic jerking and high prevalence of focal abnormalities on the EEG. Precipitation of fits by alcohol and sleep deprivation may not be recognised by the physician as part of the syndrome of JME. Diagnosis may also be delayed in patients whose absence and generalised tonicclonic seizures pre-date myoclonic jerks.
\end{abstract}

Juvenile myoclonic epilepsy (JME) was first described by Herpin in $1867^{1}$ from observations on a doctor's son, but it was not until 1957 that Janz and Mathes described the clinical and EEG features of the disease. ${ }^{2}$ The following features distinguish the disease from other seizure disorders: $:^{3-6}$ 1) Typical absences, myoclonic jerks and generalised tonic-clonic seizures in a characteristic age-related sequence, absences and myoclonic jerks usually pre-dating generalised tonic-clinic seizures; 2) Circadian distribution of myoclonic jerks (on awakening) and characteristic seizureprecipitating factors such as sleep deprivation, alcohol consumption and flickering lights; 3) Normal physical examination and CT brain scan; 4) Characteristic EEG with generalised multiple spikes and slow waves; 5) Complete remission of seizures in over $80 \%$ of patients treated with appropriate anticonvulsants (for example, sodium valproate, clonazepam); 6) Family history of epilepsy.

Recent publications suggest that the diag- nosis is frequently missed worldwide. ${ }^{3-5}$ We undertook a study of referrals to a new epilepsy clinic at St Thomas' Hospital to estimate the prevalence of the syndrome, the delay in diagnosis and the factors responsible for such delay. By studying the case records we also analysed the adverse consequences of diagnostic delay.

\section{Methods}

Consecutive patients referred between April 1989 and December 1990 to a new epilepsy clinic at St Thomas' Hospital were studied. Diagnosis of JME was established using criteria previously published. ${ }^{6}$ Patients were referred from accident and emergency departments, general practitioners in the South East Thames area, and physicians and neurologists within the hospital. Patients were clinically evaluated by one of us (CPP) to establish the diagnosis. Treatment was optimised and after appropriate follow up, case notes were independently reviewed to establish, where obtainable, the following: 1) Age at onset of absences, myoclonic jerks and generalised tonic-clonic seizures; 2) Age at diagnosis, delay in diagnosis and speciality of referring physician; 3) Factors contributing to delay in diagnosis; 4) Abnormalities on electroencephalography; 5) Treatment from presentation to diagnosis; 6) Control of seizures throughout treatment; 7) Adverse effects of delayed diagnosis.

\section{Results}

Of 180 consecutive referrals, 15 definite cases of JME were identified-a prevalence of $8.3 \%$. All patients had generalised tonic-clonic seizures and myoclonic jerks. Six of the 15 (40\%) had clinical absences. Seizure-precipitating factors, in order of importance, were fatigue, sleep deprivation, alcohol, flickering lights and stress. Five of the 15 had a first or second degree relative with epilepsy.

Absences began at a mean age of 9.5 years (range 7-13 years), myoclonic jerks at $14 \cdot 3$ years (range 10-19 years) and generalised tonic-clonic seizures at $16 \cdot 1$ years (range 9-14 years). Age at diagnosis of JME was $27 \cdot 7$ years (range 13-41 years), representing a delay in diagnosis of 14.5 years (range 1-33 years). Diagnoses on referral were usually vague and non-syndromic. They included "epilepsy" (five patients), "grand mal" (three patients), temporal lobe epilepsy (two patients), photo- 
convulsive epilepsy (one patient), TV epilepsy (one patient) and epilepsy with "temporal lobe features" ( one patient), or "focal EEG abnormalities" (one patient). One patient was referred with a putative diagnosis of JME. Other diagnoses in the notes included "pseudoseizures", "petit mal", "alcohol-induced seizures" "low seizure threshold" and "episodes of hyperventilation". Eleven of the 15 patients had consulted at least one neurologist about their epilepsy.

The factors responsible for the delayed diagnosis of JME were often multiple. In many cases the physician seemed to be unaware of the syndrome, accurately recording the syndromic features in the notes without making the diagnosis. The following extracts from a patient's diary were found in the hospital notes dated fifteen years before diagnosis: "Lots of blanks and jerks; then I had a grand mal . . . I usually have fits when rushing after getting up; usually does not happen later in the day". She was diagnosed as suffering from complex partial seizures because of focal abnormalities on the EEG (figure 1) and a sensation of fear during her absence seizures.

In some cases the history obtained from the patient was inadequate, such as, patients not being questioned specifically about myoclonic jerks. Even when asked, some patients denied myoclonic jerking which was frequently described as "clumsiness" or was interpreted as shaking related to alcohol indulgence the night before. In one of our patients generalised tonic-clonic seizures occurring after alcohol

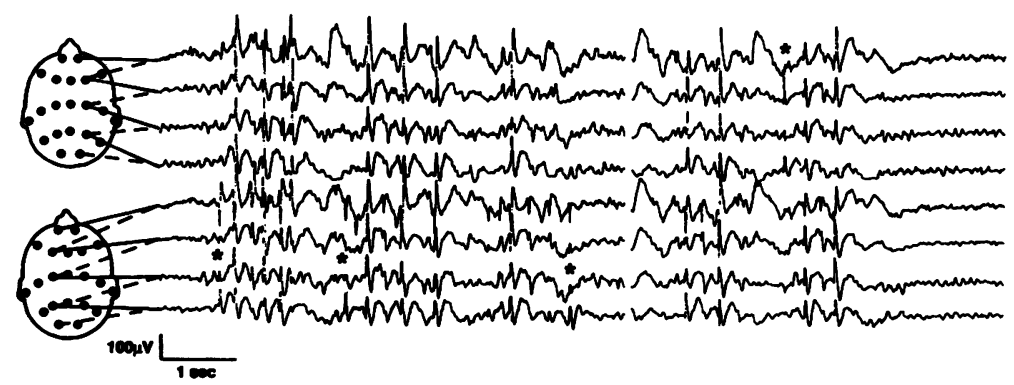

Figure 1 EEG of patient 3. Note fragmentations of continuity and of frequency of the discharges as well as focal spikes independently on left and right $\left({ }^{\star}\right)$.

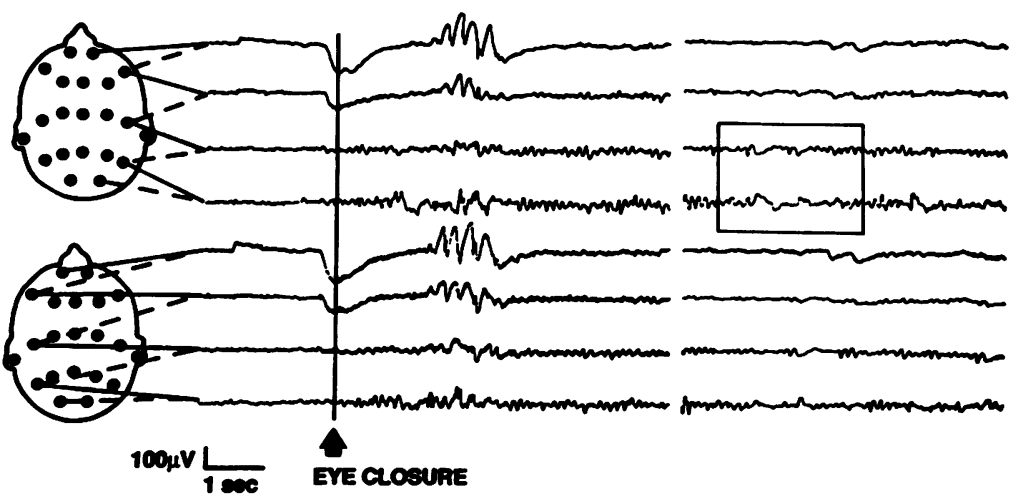

Figure 2 EEG of patient 2 (at age 30 years) showing generalised spike and wave following eye closure (the patient was not photosensitive). Previous EEGs at another hospital were reported to show posterior slow waves on the right, compatible with the diagnosis of complex partial seizures. The best example we found of such abnormalities is were interpreted as alcohol-induced.

Four of the 15 patients were misdiagnosed as having focal epilepsy, absence seizures being misinterpreted as complex partial seizures. Absence seizures in some patients were clinically inconspicuous, the patient describing feeling momentarily "cut off" or "slowed down". In at least one patient frequent absence seizures caused severe difficulties at work.

EEG was frequently entirely normal in patients on appropriate medication. Analysis of all available EEGs of all patients revealed the typical spike or multiple spike and slow wave discharges (figure 1 ) in $14 / 15$ patients. ${ }^{6}$ One untreated patient had a normal EEG but showed spike and slow wave discharge after partial sleep deprivation. Focal abnormalities, were found in $5 / 15$ patients $(33 \%)$ and consisted of transients of localised spikes, slow and sharp waves. Focal spikes were either independent or associated with the generalised paroxysms (figure 1). The same patient could have spikes in multiple locations in the same or series of EEGs. There was one patient with consistent posterior slow waves on the right which were interpreted in another hospital as compatible with complex partial seizures (figure 2). Photoconvulsive responses were obtained in $5 / 15$ patients (33\%).

Drug treatment on referral was unsatisfactory. Six patients $(40 \%)$ had never been prescribed sodium valproate. Carbamazepine was prescribed in five patients $(33 \%)$ and as monotherapy in three $(20 \%)$. It controlled seizures poorly, one patient developing myoclonic status on this drug. Five patients (33\%) were on sodium valproate monotherapy but not necessarily in appropriate dose or combination with other drugs to provide optimal seizure control. Medication had been reduced or withdrawn with adverse effects in six patients, sodium valproate from five patients (two during pregnancy, three during periods of good seizure control) and phenobarbitone from one. Adverse effects of poor seizure control included a burn requiring skin grafting, depressive illness, prohibition from driving and learning and behaviour problems.

Following optimisation of drug regimes, myoclonic jerks improved or disappeared in 13/15 patients. Improved control of generalised tonic-clonic seizures was less striking, being shown in $8 / 15$, but in the three patients who had more than two generalised tonicclonic seizures per week seizure control was excellent. Improvement occurred in $4 / 6$ patients with clinical absences. Five of the 15 patients became seizure-free. No patient deteriorated after diagnosis, though one did not attend for adequate follow up.

\section{Discussion}

We have demonstrated that JME is a common idiopathic generalised epilepsy in this population. The prevalence reported here is similar to that described in other countries. ${ }^{3-5}$ Misdiagnosis of the syndrome shown in this report has also been reported in Germany, the USA and Saudi Arabia. ${ }^{3-5}$ As many of the patients 
had consulted several physicians in and around London, including neurologists at tertiary referral centres, we believe that such misdiagnosis may be widespread in the United Kingdom.

Accurate diagnosis of JME is important for short and long term therapeutic decisions. The most effective anticonvulsant in this condition is sodium valproate. ${ }^{46}$ If patients remain symptomatic after optimisation of the dose of sodium valproate, the addition of clonazepam usually improves fit control. ${ }^{6}$ Clonazepam should not be used alone as it may precipitate generalised tonic-clonic seizures. ${ }^{6}$ Second line drugs include phenobarbitone and probably phenytoin, though carbamazepine is not effective. Treatment usually needs to be lifelong, and withdrawal of medication even in well controlled patients may precipitate seizures. This also applies to women considering pregnancy. Patients may also be warned of seizure precipitants and possible genetic risk.

We identified several factors contributing to the high rate of misdiagnosis. Physicians may not appreciate the significance or high prevalence of the syndrome, and a history of myoclonic jerks may not be elicited, may be denied by the patient or interpreted as "nervousness" or "shakes" in the morning. Absences in our patients were often misinterpreted by physicians as complex partial seizures, the frequency of focal abnormalities in the EEGs (see figures) reinforcing the erroneous diagnosis. Diagnosis is difficult in patients with absences or generalised tonic clonic seizures pre-dating the appearance of myoclonic jerks.

The typical EEG features ${ }^{6}$ of the syndrome (for example, fragmentation of the paroxysmal discharges, multiple spike and slow wave complexes and variable relationship between spike or multiple spike and slow wave) were seen in all patients' EEGs, though EEGs of patients on appropriate medication were often normal and sleep deprivation was required in one patient to elicit them. Focal abnormalities were seen in $33 \%$ of our patients and may mislead the physician into making a diagnosis of partial epilepsy with secondary generalisation. Such misdiagnosis resulted in inappropriate treatment of patients with carbamazepine.

Failure to diagnose JME not only increases patient morbidity but, because of its distinct prognosis and treatment, also confounds the analysis of trials conducted on inadequately categorised epileptic populations. To overcome these problems, we suggest that: 1) A syndromic classification of epilepsy be recorded for every patient and regularly reviewed on follow up, especially if fits are poorly controlled; 2) A history of myoclonic jerks, morning clumsiness, shaking or nervousness, be rigorously sought for every patient, and; 3) The diagnosis of partial seizures, especially temporal lobe epilepsy, be reconsidered if the EEG shows photosensitivity or generalised spike/multiple spike slow wave discharges, even in the presence of focal abnormalities.

We thank the Special Trustees of St Thomas' Hospital and British Telecom Charities Aid Foundation for their financial support of our research.

1 Herpin TH. Des Accès incomplets d'epilepsie. Paris: Ballière, 1867

2 Janz D, Mathes A. Die propulsiv-petit-mal-epilepsie. Klinic und Verlauf der sog. Blitz-, Nick-, und Salaamkrampfe. Basel: Karger, 1955.

3 Janz D. Epilepsy with impulsive petit mal (juvenile myoclonic epilepsy). Acta Neurol Scand 1985;72:339-59.

4 Delguado-Escueta AV, Enrile-Bacsal FE. Juvenile myoclonic epilepsy of Janz. Neurology (NY) 1984;34:285-94.

5 Obeid T, Panayiotopoulos CP. Juvenile myoclonic epilepsy: a study in Saudi Arabia. Epilepsia 1989;30:603-6.

6 Panayiotopoulos CP, Obeid T, Waheed G. Absences in juvenile myoclonic epilepsy: a video-electroencephalographic study. Ann Neurol 1989;25:391-7. 